\title{
Entre progreso y desarrollo La protección de la infancia en el imaginario interamericano del siglo XX
}

\section{Soledad Rojas Novoa ${ }^{1}$}

${ }_{1}$ CONICET. Universidad de Buenos Aires. Buenos Aires, Argentina. Correo electrónico: soledad.rojasnovoa@gmail.com
Recibido:

15 de mayo de 2019 Aceptado:

11 de octubre de 2019

doi: 10.34096/runa.v40i2.6034

\section{Palabras clave}

Protección social; Infancia; Familia; Interamericanismo: Organismo internacional

\section{Between Development and Progress. Protecting Childhood in the Inter-American Imaginary of the 20th-century}

\begin{abstract}
My aim in this article is to demonstrate the centrality of ideas of development and progress in articulating the social imaginaries that were paramount to conceptions of childhood in the Americas during the 20-century. I will discuss, through a historically-minded socio-anthropological lens, the emergence of childhood as a social problem in the Americas, taking the trajectory of the InterAmerican Children's Institute between 1916 and 1989 as my case-study. My argument is that the Institute and the ideologies of development and progress have strongly supported each other, as part of a process of mutual constitution that is itself an important element in the production of a widespread concern over childhood in the period.
\end{abstract}

\section{Key words}

Social protection; Childhood; Family; Inter-Americanism; International organism 


\section{Progresso e desenvolvimento. A proteção da infância nele imaginário inter-americanista do século $\mathrm{XX}$}

\section{Resumo}

Palavras-chave

Proteção social; Infância; Família; Interamericanismo; Organização Internacional
Neste artigo busco mostrar a centralidade que os imaginários de progresso e desenvolvimento alcançam na articulação dos projetos de sociedade que giraram em torno das noções de infantil na América ao longo do século XX. Para isso me dedico a uma análise socio-antropológica na chave histórica dos processos através dos quais a proteção da infância se tornou um problema social na América, tomando como prisma a trajetória do Instituto Interamericano del Niño, la Niña y Adolescentes entre 1916 e 1989. A hipótese central deste trabalho é que o circuito, cujo cerne são o Instituto e os imaginários de progresso e desenvolvimento, se sustenta em um processo de constituição mútua que forma parte dos processos de produção e difusão da preocupação social pela infância na região nesse período.

\section{Introducción}

La retórica contemporánea de derechos de niños y niñas, así como las prácticas que le son acordadas, son un producto -ni acabado ni completo- de largos procesos en los que una diversidad de actores e instituciones reclamaron legitimidad para intervenir, y disputaron prácticas y sentidos en relación con lo han entendido como una "infancia deseable" en distintos momentos históricos (Villalta, 2013). Para situar estos procesos me he interesado por el muy poco explorado caso del Instituto Interamericano del Niño, la Niña y Adolescentes, el cual, a mi juicio, resulta un mirador fructífero para indagar en la multiplicidad de ejercicios de codificación que hicieron de la protección de la infancia un problema social en América a lo largo del siglo XX.

En concreto, mi propuesta es pensar la trayectoria de este organismo como una arena de debate político-disciplinaria sobre la cual se construyeron categorías específicas para definir a ciertos niños y niñas como necesitados de protección. Sobre esa base, en este trabajo busco dar cuenta de la estrecha relación que existe entre las estructuras institucionales, la producción de conocimiento y los afanes de transformación social y, a partir de ahí, mostrar la centralidad que alcanzan los idearios de progreso y desarrollo en la articulación de los proyectos de sociedad que gravitaron sobre las concepciones de lo infantil en la región durante el siglo XX.

se basa en una investigación mayor, mi tesis doctoral titulada "La protección de la Infancia en América: una problematización histórica del presente. El caso del Instituto Interamericano del Niño, la Niña y Adolescentes (19161989)", realizada en el Instituto de Ciencias Antropológicas de la Universidad de Buenos Aires y el Laboratorio de Cambio Social y Político de la Universidad de Paris VII. Esta investigación tuvo como objetivo comprender los procesos de construcción de la protección de la infancia como problema social en América a partir del caso del Instituto, indagando en los componentes institucionales, disciplinarios y políticos de su gestión.
Para ello, organizo este texto en dos partes. En un primer momento, describo algunas particularidades del Instituto como caso de estudio, caracterizando brevemente su trayectoria y dando cuenta de algunos aspectos teórico-metodológicos que sustentan el análisis - periodización, fuentes, perspectiva-- ${ }^{1}$ En un segundo momento desarrollo la hipótesis de que el circuito nucleado por el instituto y el ideario de progreso y desarrollo funcionaron como producto e instrumento recíproca y respectivamente, lo cual configuró un espacio de problematización específico para la entendida "infancia americana". El interés de fondo es aportar nuevos elementos para la comprensión del arraigo profundo y complejo de las prácticas y representaciones que hacen a los debates 
actuales y así encontrar nuevas continuidades y rupturas en los modos de problematizar la infancia en la región.

\section{El Instituto Interamericano del Niño, la Niña y Adolescentes: un caso de estudio}

El Instituto Interamericano del Niño, la Niña y Adolescentes es el actual organismo especializado en infancia del sistema interamericano. Fue fundado en 1927 en Montevideo como un centro de estudio, documentación, consulta y propaganda dedicado específicamente a los saberes sobre la infancia en América. Surgió como iniciativa de un grupo de científicos y profesionales de distintos países de la región que, si bien pertenecían a espacios ideológicos y disciplinarios diversos, consideraron necesaria su coordinación para generar un "mapa de conocimiento" sobre la "infancia americana". Fundado por representantes de diez Estados americanos, ${ }^{2}$ la principal preocupación durante los primeros años fue motorizar el intercambio de conocimiento en torno a los problemas que venían configurándose en sus diversas realidades locales, así como a las medidas que se tomaban para hacerles frente.

Esta hipótesis fundacional y movilizadora de la gestión del instituto -según la cual la producción de conocimiento sería la herramienta fundamental para generar transformaciones duraderas en las condiciones de vida de niños y niñas- perdió buena parte de su protagonismo a partir del proceso de anexión del instituto como organismo especializado de la Organización de Estados Americanos (OEA). El acuerdo entre ambas instituciones tuvo lugar en 1949, desviando al Instituto de su rol de vanguardia en la producción y difusión de conocimiento hacia nuevas tareas, ahora directamente condicionadas por su contrato como organismo técnico. Digamos que su trabajo de definir prioridades y objetivos de acción fue sobrepasado por la tarea de generar instrumentos técnicos para abordarlos, desarrollando desde entonces asistencia especializada a los Estados, en la forma de asesorías, capacitaciones, seminarios, talleres y encuestas.

Por supuesto, este corte entre un antes y un después de la anexión a la OEA no fue categórico ni transparente. Ubicarlo es más bien una estrategia para organizar analíticamente los matices que adquiere el proceso de emergencia y estabilización del Instituto como circuito de visiones dominantes en la región, y así poder orientar la mirada hacia los condicionantes sociohistóricos de los diversos proyectos de infancia que fueron diseñados en el marco de su gestión. Esto es relevante pues aquí no interesa evaluar si las prácticas y producciones impulsadas por el Instituto han sido efectivas o eficaces en términos de sus propios objetivos, sino tomar la trayectoria de este organismo como una arena de disputas a partir de la cual sea posible conocer algunos de los debates en los que históricamente se definió a determinados niños y niñas como necesitados de protección, y así describir los procesos de institucionalización de tales definiciones.

Sobre esa base, el horizonte de este trabajo es aportar a la comprensión de los mecanismos y las pretensiones a partir de los cuales la infancia se ha tornado inteligible y, por tanto, gobernable. Parto del supuesto de que la compleja conexión de procesos históricos que componen y sostienen la trayectoria del Instituto son "huellas de formulaciones pasadas" (Aguilar, 2014), susceptibles de ser rastreadas con el objetivo de comprender en qué medida y de qué maneras ellas han pervivido, resignificadas y resituadas, y así desplegar un
2. Argentina, Bolivia, Brasil, Chile, Cuba, Ecuador, EEUU, Perú, Uruguay y Venezuela. 
3. Para los desarrollos que siguen puede resultar esclarecedor mencionar que, en el marco de la investigación mayor de la cual se desprende este artículo, el periodo de estudio (1916-1989) ha sido dividido en cuatro subperiodos: desde el Primer Congreso Panamericano del Niño, celebrado en 1916, considero como antecedentes los primeros anudamientos que hicieron de la protección de la infancia un objeto de interés para ciertos grupos en América, y en cuyos intersticios se anidaron las condiciones de posibilidad para

la fundación del Instituto en 1927. Entonces comienza un segundo subperíodo, caracterizado por los primeros esfuerzos de estabilización del Instituto y que entiendo como su período autónomo, en la medida que abarca tanto las decisiones institucionales como las prioridades programáticas que se establecen antes de firmar el acuerdo de anexión a la OEA, en 1949. La posición del Instituto como organismo especializado del sistema interamericano inaugura un nuevo modelo de gestión, determinado por la expansión y tecnificación de sus responsabilidades, redes y objetivos. El cuarto subperíodo coincide con la década de 1980, caracterizada por la acción

del Instituto frente a la masiva crisis económica en la región, cuyas particularidades imprimieron profundas transformaciones institucionales y programáticas, y termina en 1989, con la aprobación de la CIDN.

4. El consejo directivo se reunió por primera vez el 9 de junio de 1927, como adelantaba, inicialmente constituido por los representantes de diez países. Todos ellos eran varones $y$, salvo el representante peruano y el norteamericano, todos eran médicos. En 1949 se alcanzó total representación de los Estados americanos, y el consejo, paulatinamente, incluyó otras disciplinas y eventualmente mujeres; además, exigió que se tratara de expertos de ámbitos relacionados con la infancia.

5. En junio de 2016 hubo un relanzamiento de la revista en una línea editorial similar a la inicial y con tiraje de dos números por año. Disponibles en: http:// iin.oea.org/boletines.html abanico amplio de modos de problematizar la infancia. Así, desde la perspectiva que ha propuesto Robert Castel $(1994,1997)$, se trata de una problematización histórica del presente, considerando que el presente no es solamente lo contemporáneo, sino más bien un "efecto de herencia" y el resultado de una serie de transformaciones que habría que reconstruir para reactivar la carga de pasado presente en el presente.

Tal ejercicio ha requerido del análisis de un período extenso, que hiciera posible visualizar el contexto de producción y reformulación de prácticas y categorías específicas, así como los conflictos de poder y los intereses pragmáticos y morales que conducen de uno a otro. Sobre esa base, el presente del análisis ha sido el "régimen" (Pupavac, 2001) inaugurado con la aprobación de la Convención Internacional de los Derechos del Niño (CIDN), en 1989. Específicamente, se trata de las certezas que han hecho de dicho régimen una suerte de talismán que naturaliza la idea de que los derechos de niños y niñas tienen que defenderse de acuerdo con ciertos parámetros que dirimen lo deseable, y que plantean, por ejemplo, la existencia de un "interés superior del niño" o de determinadas "habilidades parentales". Si este es el punto de llegada, el punto de partida es el I Congreso Panamericano del Niño (CPN), celebrado en Buenos Aires en 1916. Tal como han planteado algunos autores (Guy, 1998; Netto Nunes, 2011), en dicho congreso se materializaron las primeras articulaciones de un "movimiento americano" preocupado por la infancia, en cuyo seno se visualizó y promovió la fundación del instituto. ${ }^{3}$

Es necesario también señalar que este trabajo se basa en el relevamiento, descripción y análisis de documentos constitutivos del instituto, principalmente, las actas de las reuniones de su consejo directivo y sus boletines de difusión. Las actas son el registro de las reuniones anuales celebradas por los representantes de cada Estado americano que forma parte del instituto. Estos representantes son el eslabón principal entre el organismo y los Estados, y responsables de los intercambios entre ambas instancias. Ellos formulan la política general del instituto y supervisan el cumplimiento de las responsabilidades de su dirección general. ${ }^{4} \mathrm{El}$ acta es redactada por el director de turno, por lo que las plumas son bien diversas en cada momento histórico. Lo que tienen en común es condensar un "deber ser" de la institución, y sus transformaciones son particularmente significativas para conocer los procesos de burocratización, expansión y complejización que ella transitó. Por su parte, el boletín es su medio de difusión regular y tiene como objetivo central la divulgación de la amplia variedad de información sobre la infancia que el organismo se encarga de recopilar - principalmente respecto de salud, acción social, educación-, así como de las "misiones" que realiza en los diversos contextos nacionales, y de las reuniones y congresos a las cuales sus miembros asisten. El primer boletín se editó el mismo año de la fundación del instituto y, con algunas lagunas importantes en los años 1990-2000, se publica aún en la actualidad. ${ }^{5}$ Durante el periodo de estudio, estaba dirigido principalmente a los pares de quienes escribían -científicos, políticos y profesionales diversos-, entre quienes circulaba a modo de intercambio con otras revistas o como donación por intermedio de los representantes de los Estados que debían difundirlo en las instituciones pertinentes, haciéndolos llegar a esos pares y procurando hacer eco de los debates que en él circulaban.

En línea con la perspectiva metodológica que mencionaba, el trabajo con estos documentos no consiste en reproducir literalmente las versiones del mundo que ellos ofrecen, tampoco en buscar una verdad que los trascienda, sino en conocer sus particularidades como producciones sociales y como campos de 
tensión. Si bien su contenido ha sido central para el análisis -y en lo que sigue me encargo de traer algunas expresiones que ayudan a visualizar el imaginario que convoca a este trabajo-, no se trata de una metodología extractiva, sino de un intento por reponer las redes de sentido construidas al momento de su creación, concibiéndolos sobre todo como herramientas dentro de un proyecto. Esto quiere decir que el material que albergan los documentos son considerados como discursos prescriptivos, no como un reflejo de las prácticas de cuidado familiares o de las prácticas de gobierno, sino como modelos que permiten vislumbrar un determinado deber ser respecto de las infancias, así como de sus modos de clasificación e intervención. En este sentido, se trata de un análisis de artefactos de un proyecto que -dejando en suspenso la pregunta por las ramificaciones de esos mandatos y cómo ellos se vieron actualizados en diversos financiamientos, servicios, instituciones o políticas en los Estados americanos- ofrece una mirada singular de los mecanismos de clasificación y administración de la infancia presentes en los debates sobre bienestar y desigualdad que tuvieron lugar en el siglo XX en la región, así como de los diagnósticos y soluciones que, en ese contexto, han interrogado la cuestión social desde el campo de lo infantil.

\section{La protección de la infancia en América, entre progreso y desarrollo}

Un análisis socioantropológico en clave histórica de la trayectoria del instituto permite afirmar que este organismo no aglutinó una mera sumatoria de individuos consagrados a un tema particular, sino que fue un entramado complejo de científicos, políticos y profesionales que configuraron un espacio estructurado por relaciones de fuerza, con intereses diversos y posibilidades de impacto también variables, cuyo principal vector era el afán de transformación social. Sobre esta base, sostengo que el potencial del instituto como caso de estudio radica en las posibilidades que ofrece para conocer y comprender la centralidad que alcanzan los idearios de progreso y desarrollo en la articulación de los proyectos de sociedad que gravitaron sobre las concepciones de lo infantil en la región durante buena parte del siglo XX.

Desde esta perspectiva, en lo que sigue, desarrollo la hipótesis de que el circuito nucleado por el instituto y el ideario de progreso y desarrollo se sostienen en un proceso de mutua constitución, es decir, que funcionaron como producto e instrumento recíproca y respectivamente. Esto puede observarse en la imbricación de tres dimensiones complementarias: en primer lugar, porque instituto e ideario vislumbran en la producción internacional de conocimiento un motor para las transformaciones sociales perdurables; en segundo lugar, porque instituto e ideario comparten una red de relaciones que proyecta sus objetivos y canaliza sus esfuerzos en la estabilización y expansión del sistema interamericano; en tercer lugar, porque instituto e ideario han abonado a los procesos de naturalización y jerarquización de la familia nuclear como pieza estratégica y necesaria para generar sistemas de orden social. Estas tres dimensiones son diferenciables solo en términos analíticos, tal como puede observarse a continuación.

\section{Producción de conocimiento y transformación social}

Históricamente, la producción internacional de conocimiento ha sido un núcleo de poder para el ideario de progreso y desarrollo en América. En ella se funda la promesa de un ascenso gradual, continuo y unidireccional hacia versiones 
"modernas" de los proyectos de sociedad en la región: el desarrollo de fuerzas productivas, el auge económico, los frutos de los esfuerzos pedagógicos, la luz de la razón irradiando sobre las conductas humanas, el perfeccionamiento de los estándares morales.

Este proyecto entendido como emancipatorio fue el faro de la gestión del instituto desde sus inicios. Es por ello que uno de sus objetivos fundacionales fue generar sus propias estrategias para producir ese núcleo de poder y así erigirse en actor de ese proceso. Como había adelantado, diversos grupos motorizaron un circuito de intercambio de prácticas y representaciones a partir del cual fuese posible justamente generar un "mapa de conocimiento" sobre la infancia en la región. Lo que interesa en este apartado es problematizar las particularidades de ese mapa, el cual, como veremos en lo que sigue, configura tres versiones posibles del conocimiento: especializado, transnacional y tecnocrático.

\section{Conocimiento especializado}

Lo primero que hay que considerar es que el Instituto fue fundado a fines de la década de 1920 en el espacio habilitado por una serie de procesos de secularización, cientifización y profesionalización concomitantes a las demandas de mayor presencia estatal y a los esfuerzos por alcanzar el progreso en la región. En ese contexto, su gestión formó parte del proceso que hizo de la preocupación social por la infancia un distintivo de las sociedades "modernas y civilizadas", y estimuló el interés general relativo a una mejor legislación y ejecución a nivel estatal en la materia o, como diría Kriste Lindenmeyer (2012), una nueva "ideología sobre la responsabilidad".

En ese contexto, desde su puesta en marche, en todo debate regional e internacional que tratara temáticas pertinentes -mas no fuese tangencialmente-, los representantes del Instituto disputaron el predominio de la infancia como una problemática autónoma. Desde los primeros congresos de medicina cuando la pediatría aún no se perfilaba como un campo autónomo-, hasta las grandes conferencias en temas de desarrollo cuando la infancia no figuraba como variable en la agenda política, estos agentes llevaron adelante verdaderas peregrinaciones con el fin de disputar espacios para esta parcela del conocimiento; buscaron materializar su presencia en diversas asociaciones, juntas, departamentos o agencias; disputaron representatividad y protagonismo en espacios académicos; coordinaron diversas publicaciones para hacer circular su trabajo; inmortalizaron sus contribuciones en archivos y bibliotecas. En definitiva, se trata de una multiplicidad de prácticas que hacen a la dimensión material del campo complejo y diverso que configuraron los saberes sobre la infancia a lo largo del siglo XX en la región.

Dicho campo generó categorías, clasificaciones e intervenciones, con reglas y metodologías propias, así como una red de actores, saberes y disciplinas mediante las cuales se buscó asegurar su hegemonía. De esa manera, con el desarrollo y sedimentación de áreas especializadas del conocimiento se desplegó progresivamente una determinada "legitimidad tecnocrática" (Lenoir, 2008), es decir, una acumulación de legitimidades técnicas, científicas, políticas y morales, que sirvieron para validar ciertas voces consideradas "expertas" (Dubois, Mohib, Oget, Schenk y Sonntag, 2005). A ellas se les supuso la posesión simultánea del saber y del saber-hacer, en el sentido de que se las confrontó a la experiencia de construir y resolver los problemas sociales relacionados 
con la infancia. El dispositivo que estas voces constituyeron históricamente configuró, en esa lógica, una legitimidad específica del gobierno de las infancias, al mismo tiempo que abasteció de las herramientas para su ejercicio.

\section{Conocimiento transnacional.}

En segundo lugar, hay que tener en cuenta el protagonismo que tuvo entre los objetivos fundacionales del instituto la preocupación por generar un conocimiento expansivo. La dispersión de proyectos nacionales en la región se concibió como un impedimento para los avances hacia el anhelado progreso. Dotar al "Nuevo Mundo" de redes de circulación y producción conjunta de ideas, así como fomentar e inventar nuevos circuitos de comunicación, fueron entonces algunas de las formas imaginadas para concretar los cambios en dirección a ese nuevo porvenir. Se trató específicamente de una serie de esfuerzos por fomentar una percepción regional transnacionalizada que se basara en la aproximación recíproca de las experiencias, prácticas y representaciones en torno a la infancia, que de manera discontinua comenzaban a emerger en el continente.

Este impulso no fue un hecho aislado en el plano internacional. Algunas iniciativas ya habían emergido en Europa incluso antes de la Primera Guerra mundial, cuyas devastadoras consecuencias fueron el motor decisivo para la estabilización de una preocupación internacional por la infancia. Se tejió entonces una serie de alianzas reformadoras que -con distintos grados de intención y con posibilidades de influenciar las orientaciones nacionales e internacionales, también diversas- se comprometieron a proteger a los miembros más jóvenes de la sociedad. ${ }^{6}$ Así, ya sea como una preocupación suscitada por el entendido "atraso" y la miseria, en el caso americano; ya sea motivado por las urgencias de la devastación producida por la guerra, en el caso internacional, estos movimientos se articularon como una medida destinada a compensar los diversos modos de violencia ejercidos sobre niños y niñas.

Bajo ese impulso, la prioridad del instituto fue llevar adelante una producción de saber conjunta entre los Estados americanos y, sobre todo, provocar y facilitar una colaboración entre ellos. Esta preocupación movilizó en gran medida su gestión -en particular, las acciones dirigidas a fomentar circuitos y redes de comunicación, así como a capitalizar un encuentro y una aproximación recíproca de experiencias y modelos-, en un esfuerzo por aunar iniciativas que se consideraron aisladas y por revertir la práctica de "vivir ignorándose" entre expertos, como reclamó Luis Morquio (1927), primer director del instituto, en el discurso inaugural del organismo.

Entonces el organismo articuló una serie de circuitos que, si bien son sostenidos por los representantes de los Estados y las circulaciones del saber que ellos hacen posibles, sobre todo reposan en lo que podríamos pensar como el "entre-mundos" de esos individuos y esas circulaciones -usando la expresión de Renauld Payre (2011)-. En ese "entre-mundos" no cabe la idea de influencia cuando se trata de explicar la similitud de referencias, innovaciones o políticas que se van dando entre los diferentes países producto del fomento de los movimientos de ideas y personas; no se trata de relaciones binarias determinadas por principios de imitación, importación o transferencia lineal; tampoco de un préstamo de un país a otro. En pocas palabras, no importa tanto aquello que se intercambia -como sucedería en la lógica mercantil-, como el intercambio mismo y lo que de/en él se genera.
6. Varias de ellas tuvieron un rol importante en la conformación de instituciones centrales en el debate internacional, por ejemplo, el Comité de Protección de la Infancia de la Sociedad de las Naciones - predecesor de UNICEF-, en 1925. Este es un proceso muy vasto, el cual supera con creces los propósitos de este artículo. Para su revisión, considerar los interesantes trabajos de Joëlle Droux (2011) y Dominique Marshall (1999, 2008 2009). 
Esta imagen ayuda a comprender las redes particulares que estos actores encarnaron y a discernir en qué medida sus trayectorias disímiles, así como sus motivaciones y constricciones diversas, confluyeron en los procesos de producción e institucionalización del saber sobre la infancia en la región. Al mismo tiempo, permite comprender que estos desplazamientos y transacciones de conocimiento funcionaron como diásporas, siendo el instituto un centro de concentración de un saber que se va difuminando por medio de la acción de esos agentes especializados que operan de manera interna en sus contextos nacionales. Se trata de una disputa constante entre el objetivo de que el conocimiento que se produjo y circuló en ese "entre-mundos" se viera eventualmente reflejado en políticas concretas y el reconocimiento de que la protección de la infancia opera de manera histórica o geográficamente situada. Finalmente, es posible afirmar que las estrategias desplegadas en este contexto determinan y están determinadas por la tensión entre la autonomía científico-política de los actores del circuito y su capacidad de investir el campo estatal, de tal manera que, en el reverso de la expectativa de que dichos actores lograran efectos en la definición de modelos locales de protección de las infancias habita el riesgo de asumir una perspectiva universalizante de las prácticas y representaciones producidas.

\section{Conocimiento tecnocrático.}

En tercer lugar hay que tener presente que, desde su fundación, el instituto formó parte de una red de sentidos informada por criterios modernizadores hacia los cuales se orientaron masivamente los proyectos de transformación de las realidades sociales. Entonces, el futuro se pensaba en clave de un idealismo triunfante; mientras que el presente tomaba la forma de una complejidad creciente de las relaciones sociales, marcadas en el campo de lo infantil por problemáticas diversas -mortalidad, educación, orfandad y abandono, delincuencia, prácticas de circulación y traspaso de niños, alimentación y lactancia, vivienda-que se pensaba solo serían combatidas con la certeza de la ciencia. Esto es relevante pues ambas convicciones -futuro y ciencia- vectorizaron las propuestas de los actores del circuito del Instituto. La síntesis de esas propuestas puede observarse en los documentos estudiados en la forma de un "modelo preventivo" que caracterizó históricamente su prédica. Conjugando una mirada científica y social, este modelo condensó una serie de mecanismos diseñados en el esfuerzo por alejarse de las lógicas paliativas o represivas, para actuar, en cambio, mediante estrategias que controlaran el ambiente en que se desarrollan niños y niñas, con tal de poder predecir, mediante técnicas estandarizadas, su futuro. Con ello, independientemente del periodo estudiado, es posible observar una tendencia constante de los debates a ampararse y disputar los argumentos bajo el alero de la "neutralidad" del modelo científico, para presentarse como herramientas meramente técnicas, que vienen a intervenir sobre lo que se considera un "problema dado" -las infancias desprotegidas, en ambientes nocivos pero transformables-, susceptible de solución por fuera de las tensiones y contradicciones constitutivas a la sociedad, mediante métodos estandarizados.

Desde esta perspectiva, en el circuito del instituto circuló una idea de Estado cuya acción debía basarse en datos, previsiones y cálculos orientados hacia los resultados, que validaba una mirada tecnocratizada del campo de lo infantil. Así, guardando los matices, los actores de este circuito tendieron a pronunciarse más sobre la presencia de ciertas infancias necesitadas de protección que sobre las ausencias que las (re)producen. Si bien en la década de 1980 los 
factores estructurales cobraron mayor presencia en los debates, las infancias que fueron definidas como necesitadas de protección tendieron a entenderse como susceptibles de reglamentar, gestionar y controlar; mientras que las causas y formas de su existencia fueron preferibles de ocultar, disimular y opacar. Por lo mismo, la "necesidad de protección" tendió a problematizarse en un orden práctico, que solo parecía reclamar soluciones técnicas, mientras que en el reverso, el aprecio por ese saber tecnocratizado fue conformando una zona silenciada y excluida de la discusión.

Esta versión tripartita del conocimiento sobre la infancia americana en el siglo XX -especializado, transnacional y tecnocrático- permite retornar sobre la tensión histórica que, decía, sitúa al instituto entre un rol de vanguardia en la definición de problemas relacionados con la infancia y su lugar como organismo técnico del sistema interamericano, y así plantear su riqueza como terreno de análisis para discernir interesantes versiones y variaciones de la relación entre ciencia y política en el campo de la protección infantil. En efecto, en esa tensión se anida la configuración del instituto como arena de condensación de diversas disputas en torno al papel del Estado en la "administración de la infancia" (de Souza Lima, 2002; Vianna, 2002; Villalta, 2010). En este sentido, el movimiento nucleado en torno al organismo se constituye como un espacio relativamente autónomo que, al poseer un modo particular de conocimiento sobre el mundo, crea, en el mismo gesto, su propio objeto y los principios de comprensión y explicación convenientes a este objeto (Bourdieu, 1999). Dicho espacio se sostiene en las continuas negociaciones suscitadas por la implementación de estrategias de gestión en el campo de la infancia y la familia, así como en la circulación de principios hegemónicos internacionales que hacen que la recepción de los objetos que produce sea altamente heterogénea y dependiente del interés particular de las culturas locales.

\section{La materialidad de los intercambios: procesos de identificación y ramificación}

Tal como ha señalado Dominique Marshall (2008), el campo de protección de la infancia ha sido por excelencia un terreno fructífero para fomentar el encuentro entre los Estados, pues concerta un espacio común "aceptable para todos". Desde esa perspectiva, la historiadora plantea que los intentos por problematizar la infancia, en particular en los espacios transnacionalizados, han estado históricamente signados por una "diplomacia del bienestar", es decir, por un posicionamiento moral que determina los debates sobre un tema que se supone debiera generar unanimidades inmediatas. El instituto no es la excepción a esta regla, y quienes lideraron la iniciativa supusieron que ella causaría una adhesión "espontánea" por parte de los Estados americanos. Pero los documentos revisados permiten observar que, si bien las prácticas y representaciones que su gestión motorizó parecían rápidamente alcanzar circulación en este territorio "aceptable para todos", las condiciones sociales, culturales y materiales no bastaban para sostener su incipiente presencia. Hacía falta un paso más: materializar el movimiento de protección de la infancia en un marco institucional estable, construyendo un circuito dotado, al menos, de estabilidad económica y representativa.

Para construir dicha estabilidad, el establecimiento de lazos de cooperación con el sistema interamericano fue decisivo. ${ }^{7}$ Hay que considerar que la tradición interamericana antecedía varios de los esfuerzos que fueron propios del instituto - por ejemplo, el interés por recopilar, sistematizar y difundir información; el fomento de la creación de instancias especializadas; o la búsqueda
7. En términos formales, dicho sistema coincide con el histórico circuito de cooperación técnica, comercial y diplomática a nivel regional, materializado en las conferencias panamericanas -la primera, celebrada en Washington en 1889- y en diversas asociaciones estables y de gestión continua tanto la Oficina Internacional de las Repúblicas Americanas, fundada en 1902, como sus sucesoras, la Unión Panamericana (1910) y posteriormente la Organización de Estados Americanos (1948)Pero este circuito resiste múltiples análisis. Sobre las posibilidades de entender el "interamericanismo" como una manera de designar las relaciones entre EEUU y

América Latina, y como un modelo específico de progreso y desarrollo, existen diversos estudios, y su análisis excede a este trabajo. Para una versión amplia, recomiendo los trabajos compilados por Frances Fukuyama (2006). 
8. No hay que olvidar que el Instituto es el único organismo especializado de la OEA que fue anexado, es decir, que existía independientemente y que no fue fundado en relación con la OEA.

Se habían creado con anterioridad: la Organización Panamericana de la Salud (1902), la Comisión Interamericana de Mujeres (1928), el Instituto Panamericano de Geografía e Historia (1928), el Instituto Indigenista Interamericano (1940), el Instituto Interamericano de Cooperación para la Agricultura (1942). Al mismo tiempo, llama la atención que, para designar este proceso, en los documentos oficiales se ocupe la expresión "anexar”, dada la connotación violenta que geopolíticamente supone que una unidad mayor se apodera de una menor, transformando sus principios. de diálogo y conocimiento recíproco a nivel regional- y, en ese sentido, los procedimientos, redes estratégicas y formas burocráticas del modelo regional fueron fuente de inspiración para sus propias derivas. Al mismo tiempo, para el sistema interamericano resultaba deseable sumar a sus posibilidades de acción un área de la realidad social que todavía no tenía entre sus campos estratégicos, tal y como era la protección de la infancia. ${ }^{8}$ Sobre esta base, el instituto y el sistema interamericano articularon sus intercambios en un entramado fino y cotidiano de microprácticas que hicieron de cada decisión, de cada objetivo, de cada acuerdo, de cada programa, una estrategia de legitimación de la cooperación entre ambas instancias. Esto se observa de manera ejemplar en la forma y lenguaje que cobran los documentos institucionales, con particular claridad en las actas de las reuniones del consejo directivo, cuya burocratización responde en gran medida a los esfuerzos de registro y comunicación entre el instituto y el sistema interamericano, antes y después de su anexión a la OEA. Si observamos de cerca estos intercambios podemos comprender que el instituto no operó como una entidad etérea y abstracta, sino como un dispositivo bien anclado en una serie de estrategias que materializaron su existencia, su imbricación y su compromiso con el sistema regional. Al menos dos mecanismos articulan estas estrategias con claridad.

Por una parte están aquellos que entiendo como mecanismos de identificación, esto es, los esfuerzos por generar un movimiento cohesionado entre el sistema regional y el instituto sobre la base de una retórica común. Se trata en general de una retórica de los contrastes, la cual ubicó sostenidamente a ciertos comportamientos del lado de la "barbarie" y a otros del lado de la "civilización"; a unos del lado de la "catástrofe" y a otros del lado del "orden"; uno y otro siempre como opuestos irrevocables. Sobre esa base, es habitual encontrar en los artículos publicados en el boletín a las infancias necesitadas de protección y sus familias situadas del lado del "desorden" o de lo "salvaje", es decir, del lado de la naturaleza y el instinto -“vicioso", "alcohólico"-, o del lado de las costumbres resistentes -"ignorante", "prejuicioso", "reacio"-, poniendo en cuestión además su comportamiento económico -"malgasto", "derroche", "falta de previsión"- o sanitarios -“suciedad", "enfermedad", "muerte"-. En la contraparte, se esperaba que esas familias pudiesen "pasar de la ignorancia a la sensibilización", "informarse", "tomar parte activa", "llenarse de entusiasmo", "comprometerse", "participar", "colaborar", "generar sentimientos de vinculación". En la misma línea, por ejemplo, se impulsaron patrones que tienen que ver con la "disciplina", "tesón", "perseverancia", "tenacidad", "constancia", "empeño", "esfuerzo" o la idea de "la felicidad que causa el deber cumplido", en oposición a la lógica de "obtener más con menos esfuerzo" o de "exigir derechos y esquivar deberes". Esta problematización binaria que habita con insistencia las páginas del boletín del instituto tendió a homogenizar y a teñir de manera uniforme las prácticas y representaciones sociales que se traman en este imaginario interamericano. Se trata de formas discursivas, proyectos, visiones, creencias y metáforas que el Instituto y el sistema interamericano compartieron históricamente, y que informa tanto los modos de entender la acción institucional, como de clasificar las infancias y sus familias. Sobre esa base, los agentes especializados que conformaban el circuito del instituto respondieron al objetivo de vitalizar espacios de circulación de prácticas y representaciones constitutivas del sistema interamericano, con el fin de introducirlas directamente en los imaginarios políticos de los Estados americanos.

Por otra parte, este imaginario se canalizó gracias a lo que entiendo como mecanismos de ramificación, que lo difundieron y propagaron en los contextos 
locales de la región. En la primera versión del instituto -es decir, su trabajo como "centro especializado de estudio, documentación, consulta y propaganda sobre las experiencias infantiles en la región", hasta entrada la década de 1950 - primaron dos estrategias que buscaban nuclear la información producida y hacerla circular: la publicación regular de su boletín, y la organización y participación en diversos congresos y reuniones científicas, a nivel nacional, regional e internacional. Como había mencionado, el boletín es la publicación regular del instituto, cuya existencia de alguna manera materializa su objetivo de centralización, sistematización y difusión de información. En opinión de Anne Emmanuelle Birn (2006), este boletín resultó ser uno de los diarios de mayor circulación internacional por esos años, y permitió a diversos científicos e investigadores publicar y compartir sus avances. En tal sentido, esta publicación fue capaz de "hacer existir" científicamente a los participantes del circuito, ya sea validando sus aportes en relación con las categorías vigentes, o distinguiendo a algunos de ellos positivamente por una contribución reconocida por el grupo, así como también consolidando su presencia en varias latitudes, gracias a su alcance internacional. Algo similar ocurría en los diversos congresos y otros eventos de circulación científica. Ellos eran, en palabras de Birn (2006), la clave de la organización internacional en la primera mitad del siglo XX. Tal como ha problematizado Corinne Pernet (2007) para el escenario internacional, representaban un rico medio de intercambio, complementario a los escritos, que buscaba cultivar una cohesión entre científicos, intelectuales y políticos, más allá del intercambio de información. Los registros sobre estos eventos se tornan interesantes en la medida que dan cuenta de los modos en que los diversos pero circunscritos actores tomaban el lugar de "embajadores", de representantes de ciertos intereses nacionales o regionales $\mathrm{y}$, al mismo tiempo, de "especialistas", interesados en consolidar aquel espacio especializado sobre la infancia del que hablábamos. En ese sentido, los registros nos permiten conocer las particularidades de estos intercambios, que generalmente funcionaron como escenas diplomáticas o de promoción de la política extranjera, en las cuales todo transcurría de manera muy protocolar y, en tanto el interés que movilizaba a los representantes del instituto era participar decididamente del circuito, se mantienen en una posición complaciente y halagadora. Para decirlo en palabras de diversos trabajos encontrados en el boletín, estos registros nos permiten conocer discursos "del más alto valor", "conceptuosos", "hermosos", "de alta investidura", "de alta cultura", "prestigiosos", "ilustres", "inteligentes", "eminentes", "distinguidos", "merecedores de homenaje", "con autorizada opinión", "dignos", "lúcidos".

A partir de la década de 1950, estas dos estrategias -el boletín y la participación en reuniones científicas- tuvieron un protagonismo oscilante frente a la emergencia de otras actividades que tomaron fuerza en razón de los nuevos objetivos del instituto, una vez concretada su anexión a la OEA. Estas actividades respondían sobre todo a un perfil de asistencia técnica para los Estados, en el marco de los esfuerzos de cooperación horizontal y del modelo de desarrollo económico-social, los que definieron de manera determinante la acción multinacional durante la segunda mitad del siglo XX. Concretamente, se trató de diversas asesorías, capacitaciones, cursos, seminarios, talleres, encuestas, que buscaron formar a aquellos especialistas -técnicos y profesionales- que serían luego los encargados de movilizar los saberes y prácticas producidos de vuelta a sus países. Estas actividades fueron, por lo demás, coherentes con el interés de desarrollar un programa de colaboración, intercambio y producción de conocimiento, así como con la masificación de la formación profesional. 
En su conjunto, ambos mecanismos -de identificación y de ramificación-nos permiten pensar que el instituto es producto e instrumento de los procesos de expansión del sistema interamericano durante el siglo XX en la región, a la manera en que dos metáforas cognitivas básicas nos permiten graficarlo: el árbol y la onda. El árbol describe el pasaje de la unidad a la diversidad, mientras que la onda es todo lo contrario, ya que describe cómo la uniformidad engulle toda una inicial diversidad. A través del prisma del instituto, la infancia moderna puede entenderse como una onda, pero una onda que se interna en las ramas de las tradiciones locales y siempre queda transformada significativamente por ellas.

\section{Familia nuclear y orden social}

El "modelo preventivo" que decía caracterizó la prédica del Instituto a lo largo del periodo de estudio buscaba calar en la realidad de las familias, produciendo ideales, instalando valores morales, creando roles y responsabilidades contingentes. Se trata de un modelo que buscó definir "pautas adecuadas" de cuidado, crianza y socialización de niños y niñas, así como las formas de asegurar su cumplimiento, forjando imaginarios particulares respecto de los modos cotidianos de vivir, que abarcan dimensiones mucho más amplias que la protección infantil.

En línea con lo que observa Eduardo Netto Nunes (2011) para caso el de los congresos panamericanos del niño, la infancia en el circuito del instituto fue entendida como algo "germinal", "moldeable", "perfeccionable", como una hoja en blanco que había que introducir en la sociedad según determinados parámetros de deseabilidad. Por lo mismo, se la posicionó como una "promesa de futuro", es decir, una herramienta central para las transformaciones sociales. Desde esa perspectiva, la preocupación social por el bienestar infantil -digamos, el interés en que niños y niñas alcanzaran un desarrollo físico y moral dentro de lo deseable, y de que su protección significara la menor carga posible para el Estado- planteaba inmediatamente un pasaje de un tiempo a otro, del presente al futuro. Dicho de otro modo, la protección de la infancia como problema social se constituye en una vía para imaginar el futuro, incluyendo aquí los aparatos para su definición, clasificación y control. Se trata, en definitiva, de la intervención de los expertos sobre el presente infantil, buscando delimitar el futuro social, y transformando, de paso, a la infancia en una bisagra que articula pasado, presente y futuro (Carli, 2002).

Una versión de esta tensión temporal que se concentra en el circuito nucleado por el Instituto emerge si pensamos que las diversas estrategias de protección y socialización de la infancia que ahí se diseñaron no solo apuntaban a la constitución de "buenos ciudadanos" en el futuro, sino que además funcionaron como reguladores morales y materiales de las familias en el presente considerando que, al tiempo que apuntaron al "niño del futuro", crearon roles y determinaron responsabilidades contingentes, al delimitar y clasificar los comportamientos de "una buena madre" o de "un padre responsable". En esa ecuación, el futuro como forma perfecta permitía poner en evidencia las "fallas del presente": de ahí el cuestionamiento constante a los sectores populares en el cumplimiento de su función de socializar a los miembros más jóvenes; de ahí las propuestas de su entrenamiento para el ejercicio de esa función. Esto redundó en un apuntalamiento, desde diversos marcos disciplinarios, de un modelo educativo orientado a inyectar "habilidades familiares" ahí donde ellas se consideraban ausentes. Así, anidada en un imaginario de "ajuste" o 
de "evitar la desviación", se desplegó una serie de tecnologías para acompañar a las familias en ese proceso de "adaptación mutua" con la sociedad. Para ello se forjó un horizonte de máximas morales y de comportamiento, así como de expectativas e ideales, que apuntaban justamente a un gobierno de las competencias, los compromisos y los deseos, con tal de construir familias responsables con sus condiciones de vida y creativas ante la adversidad.

En un principio (1927-1949), las intervenciones se orientaron a regular los intercambios afectivos y económicos en razón de los ideales impuestos por la "familia legítima", heterosexual, con base en el matrimonio y con hijos concebidos bajo su ley. A estas estrategias las llamé tecnologías de legitimidad, y considero entre ellas la "profilaxis del abandono", caracterizada como aquellas intervenciones dirigidas a la "madre soltera", que buscan apuntalar los "desajustes" de las familias sin hombre-sustento y redireccionarlos hacia el "cauce natural" de la familia nuclear; las enseñanzas ligadas a la puericultura que fueron transmitidas en diversos contextos a las madres -hospitales, albergues, comedores-, para obtener de su parte cuidados de higiene y crianza que asegurasen el óptimo desarrollo de sus hijos/as; o la "regulación nupcial", una campaña de instrucción que buscó infundir aptitudes morales en el matrimonio, para que este se desarrollara dentro de los parámetros esperados, es decir, entre un hombre y una mujer que tienen como objetivo la procreación y que toman las medidas necesarias para que su descendencia sea saludable.

En un segundo momento (1950-1980), el foco de atención ya no estaba puesto únicamente en los miembros de la familia, sino en la familia en su conjunto, de manera que se revalorizó al grupo como primer espacio de socialización y garante de la "formación de la personalidad" de niños y niñas, y de desarrollar en ellos y ellas un "sentimiento de pertenencia" en la sociedad. Desde esta perspectiva, la familia ya no es solamente un grupo de procreación y sobrevivencia, tampoco una mera unidad moral, depositaria del honor de sus miembros, sino que es, sobre todo, un "núcleo pedagógico", pues en ella se aprenden los patrones - pensamientos, sentimientos, actitudes- de la vida individual para la vida social. En ese escenario, a partir de lo encontrado en los boletines se entiende que lo regular y lo esperado es que las familias sean capaces de "ser productivas" y de "incorporarse" al proceso de desarrollo. Una serie de tecnologías de educación para la responsabilidad fueron diseñadas en consecuencia, por ejemplo, la "educación para padres", que los entrena a responsabilizarse por el "ejercicio consciente" de la parentalidad; la "educación para la sexualidad", que entrega herramientas para la planificación familiar; o la "educación de las prácticas domésticas", que acompaña a las familias en la optimización de los recursos y la economía del hogar.

Entrada la década de 1980 es posible identificar un nuevo desplazamiento, determinado por la retórica de la "lucha contra la pobreza" que la crisis económica de esos años acuñó. Se trata de un discurso que clasifica a las familias en una doble dimensión: por un lado, determinada por el acceso a necesidades mínimas biológicas -cuantificables y con pretendida universalidad-, y por otro, al desarrollo de las capacidades para lidiar con su condición de precarización, en la lógica de una "gestión de la fragilidad humana", como lo ha explicado Robert Castel (2011). Esta perspectiva ya venía instalándose como modelo del buen gobierno de la sociedad desde la década de 1970, pero la crisis agregó nuevas exigencias. Ella puso en cuestión las funciones tradicionales asignadas a la familia, justamente en la medida que se radicalizó su inestabilidad y falta de flexibilidad frente a situaciones adversas. Al mismo tiempo, abrió nuevas dimensiones en el campo de las responsabilidades con el bienestar infantil, 
al generar lo que en el circuito del instituto se entendió como "patologías sociales", entre ellas, la violencia, el abuso, el maltrato, la explotación o la drogadicción. En sentido amplio, entre los boletines se habló de una "familia fragilizada" por los embates de la crisis, cuya función socializadora estaba ampliamente deteriorada. Como efecto causal y lineal de ese deterioro habrían emergido formas particulares de la "infancia en situación irregular".

Como vemos en las diversas versiones de este horizonte de máximas que el instituto predicó, el desafío principal de las familias fue funcionar dentro de condiciones deseables de amar y trabajar, aquellas dos dimensiones que Freud sostuvo como constitutivas del malestar en la cultura y que, en este caso, apuntaron a fomentar la reproducción, no de cualquier familia, sino de una legítima, saludable, educada y autónoma. En este sentido, la trayectoria del instituto se presenta como un prisma para observar la recíproca construcción entre la preocupación social por la infancia y la pregnancia de un imaginario familiarista que abonó a los procesos de naturalización y jerarquización de la familia nuclear como pieza estratégica para generar sistemas de orden social en los Estados americanos en el siglo XX.

\section{Comentarios finales}

Uno de los aportes de las investigaciones en clave histórica es que permiten dimensionar la profundidad de los procesos que hoy se nos revelan prístinos, así como constatar que ellos no avanzan ni impactan de forma homogénea en el campo que construyen. En este sentido, las reflexiones aquí expuestas buscan acompañar el ejercicio adelantado por investigadoras como Patrice Schuch (2012), que hace tambalear el poder que expansivamente se le ha otorgado al marco de derecho inaugurado por la CIDN, sobre el supuesto de que tal documento podría determinar una nueva y única forma para pensar la infancia, o hacer emerger "una nueva generación" de prácticas, actores y disciplinas como recambio de aquellas "contaminadas" con la antigua cultura de protección.

Como hemos visto, las tendencias a compartimentar la experiencia infantil, a universalizar representaciones irrepetibles, a tecnocratizar las intervenciones o a uniformar o a moralizar las prácticas familiares, que la CIDN busca superar, están enraizadas en imaginarios sociales tramados con tanta fuerza y hace tanto tiempo que resultarían difíciles de desarticular únicamente a partir de un documento. Por ello, es mi intención que la lectura que propongo de los procesos de mutua constitución entre este circuito y los idearios de progreso y desarrollo pueda ofrecer nuevas herramientas para interrogar las configuraciones actuales del "campo de lo infantil", particularmente para el caso de las experiencias latinoamericanas.

Sin duda, la CIDN ha introducido una novedad en el campo de la protección infantil en la región, pero de él no podría esperarse una transformación mecánica o absoluta: si bien ha permeado los imaginarios sociales sobre la infancia, la integralidad de sus principios dista mucho de tener el impacto transformador prometido. $Y$ es esperable que así sea si reconocemos el dinamismo de los procesos que busca delimitar, pues ellos son flexibles y porosos, y se sostienen inevitablemente en un delgado límite que se teje entre las legítimas intenciones de los actores que buscan procurar mejores condiciones de vida a niños y niñas, los cálculos políticos y económicos del costo y beneficio de las acciones, la función pedagógica de ellas y las prácticas de control y administración cotidianas que busca poner en marcha la cohorte de 
científicos, profesionales y políticos que sostienen los circuitos de protección. En el mismo sentido, el hecho de que la vida de niños y niñas esté constante y profundamente amenazada por las políticas de ajuste y la progresiva lejanía del rol público del Estado tampoco ha ayudado a este proceso. Por lo mismo, cuestionar el carácter supuestamente inédito de los problemas y soluciones que la CIDN suscita nos permite también tensionar los límites conceptuales y los esquemas interpretativos a partir de los cuales la infancia, la desigualdad y el rol del Estado se han pensado, interpretado y materializado. 


\section{Q Referencias bibliográficas}

"Aguilar, P. (2014). El hogar como problema y como solución. Una mirada genealógica de la domesticidad a través de las políticas sociales. Argentina 1890-1940. Buenos Aires: Ediciones del Centro Cultural de la Cooperación.

» Birn, A.-E. (2006). The national-international nexus in public health: Uruguay and the circulation of child health and welfare policies, 1890-1940. História, Ciências, Saúde-Manguinhos, 13(3), 33-64.

» Bourdieu, P. (1999). Meditaciones pascalianas. Barcelona: Anagrama.

"Carli, S. (2002). Niñez, pedagogía y política. Transformaciones de los discursos acerca de la infancia en la historia de la educación argentina entre 1880 y 1955. Buenos Aires: Miño y Dávila.

»Castel, R. (1994). “Problematization” as a mode of reading history. En J. Goldstein (Ed.), Foucault and the writing of history (pp. 237-253). Oxford: Blackwell.

» Castel, R. (1997). Présent et généalogie du présent: une approche non évolutionniste du changement. En D. Franche, S. Prokhoris e Y. Roussel (Eds.), Au risque de Foucault, (pp. 161-167). París: Centre Georges Pompidou.

» Castel, R. (2011). La gestion des risques. París: Les éditions de Minuit.

"De Souza Lima, A. (2002). Sobre gestar e gerir a desigualdade: pontos de investigação e diálogo. En A. De Souza Lima (Org.), Gestar e gerir. Estudos para uma antropologia da administracão pública no Brasil (pp. 11 a 22). Río de Janeiro: Relume-Dumará.

»Droux, J. (2011). L'internationalisation de la protection de l'enfance : acteurs, concurrences et projets transnationaux (1900-1925). Critique Internationale, 52, 17-33.

»Dubois, S., Mohib, N., Oget, D., Schenk E. y Sonntag, M. (2005). Connaissances et reconnaisance de l'expert, Les Cahiers de l'INSA de Strasbourg, 1, 89-108.

» Fukuyama. F. (Comp.) (2006). La brecha entre América Latina y Estados Unidos. Determinantes políticos e institucionales del desarrollo económico. Buenos Aires: Fondo de Cultura Económica.

» Guy, D. (1998). The Pan American Child Congresses, 1916 to 1942: Pan Americanism, Child Reform, and the Welfare State in Latin America. Journal of Family History, 23(3), 272291.

》 Lenoir. R. (2008). Famille ou mode de reproduction de la structure sociale, Regards sociologiques, 35, 111-117.

" Lindenmeyer, K. (2012). Children, the State, and the American Dream. En P. Fass (Ed.), Reinventing childhood afer World War II (pp. 84-110). Filadelfia: University of Pennsylvania Press.

»Marshall, D. (1999). The Formation of Childhood as an Object of International Relations: the Child Welfare Committee and the Declaration of Children's Rights of the League of Nations. International Journal of Children's Rights, 7(2), 103-147

» Marshall, D. (2008). Dimensions transnationales et locales de l'histoire des droits des enfants. La Société des Nations et les cultures politiques canadiennes, 1910-1960, Genèses, 71, 47-63. 
» Marshall, D. (2009). The Causes, Promises and Problems of Coordinated Actions in Favour of Children in War and Peace: Philanthropists, Experts and the League of Nations, 1914-1930. Workshop Trans-national Networks of Experts and Organizations. Ginebra.

» Morquio, L. (1927). Instituto Internacional Americano de Protección a la Infancia. Su inauguración. Boletín del Instituto Internacional Americano de Protección de la Infancia, 1(1), $29-67$.

» Netto Nunes, E. (2011). A infância como portadora do futuro: América Latina, 1916-1948. (tesis de Doctorado). Facultad de Filosofía, Letras y Ciencias Humanas, Universidad de San Pablo. San Pablo, Brasil.

" Payre, R. (2011). L'espace des circulations. La fabrique transnationale des sciences administratives (années 1910-1950). En P. Laborier, F. Audren, P. Napoli, y J. Vogel (Dirs.), Les sciences camérales. Activités pratiques et histoire des dispositifs publics (pp. 283-307). París: PUF.

» Pernet, C. (2007). Les échanges d'informations entre intellectuels: la Conférence comme outil de coopération intellectuelle à la Société des Nations. En F. Valloton (Dir.), Devant le verre d'eau: regards croisés sur la conférence comme vecteur de la vie intellectuelle 1880-1950 (pp. 91-106). Lausana: Editions Antipodes.

»Pupavac, V. (2001). Misanthropy Without Borders: The International Children's Rights Regime. Disasters - Overseas Development Institute, 25(2), 95-112.

»Schuch, P. (2012). Justice, culture and subjectivity. Vibrant - Virtual Brazilian Anthropology, $9(2), 34-69$.

»Vianna, A. (2002). Quem deve guardar as crianças? Dimensões tutelares da gestão contemporânea da infancia. En A. De Souza Lima (Org.), Gestar e gerir. Estudos para uma antropologia da administracão pública no Brasil (pp. 271- 312). Río de Janeiro: RelumeDumará.

»Villalta, C. (2010). La administración de la infancia en debate. Entre tensiones y reconfiguraciones institucionales. Estudios en Antropología Social, 1(2), 81-99.

»Villalta, C. (2013). Un campo de investigación. Las técnicas de gestión y los dispositivos jurídico-burocráticos destinados a la infancia pobre en la Argentina. Revista CIVITAS, $13(2), 245-268$. 
